
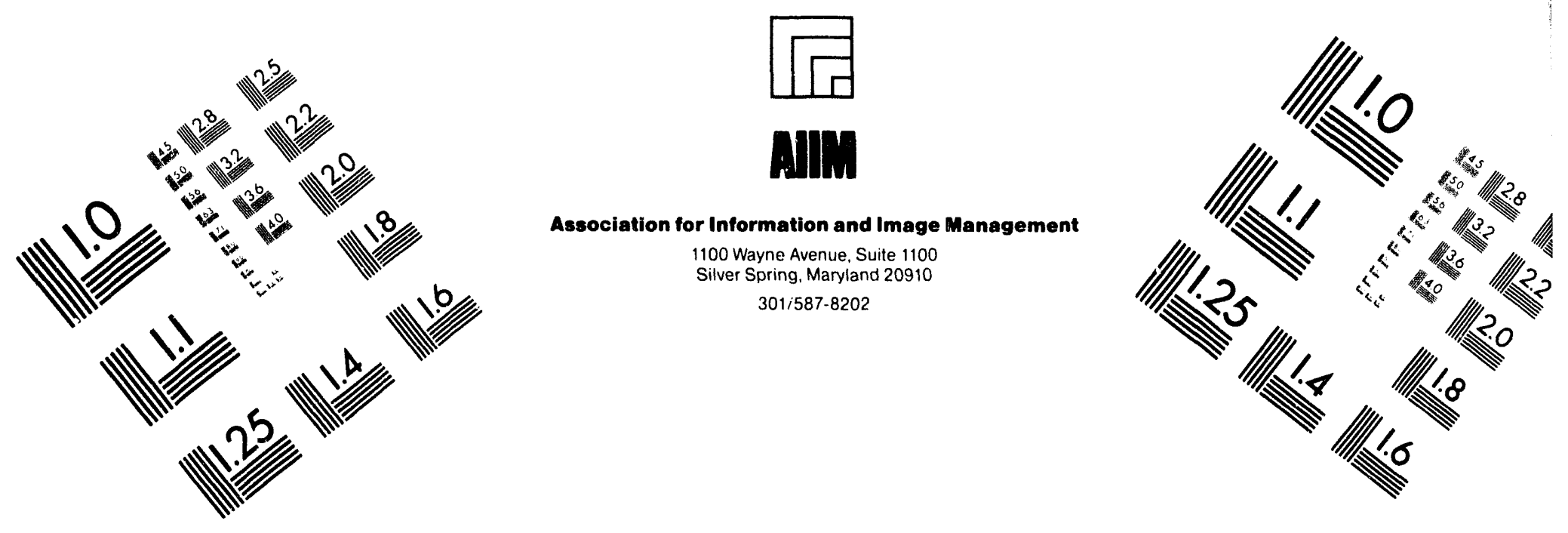

\title{
Centimeter
}



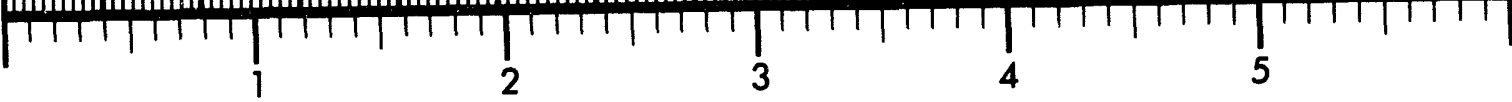
Inches
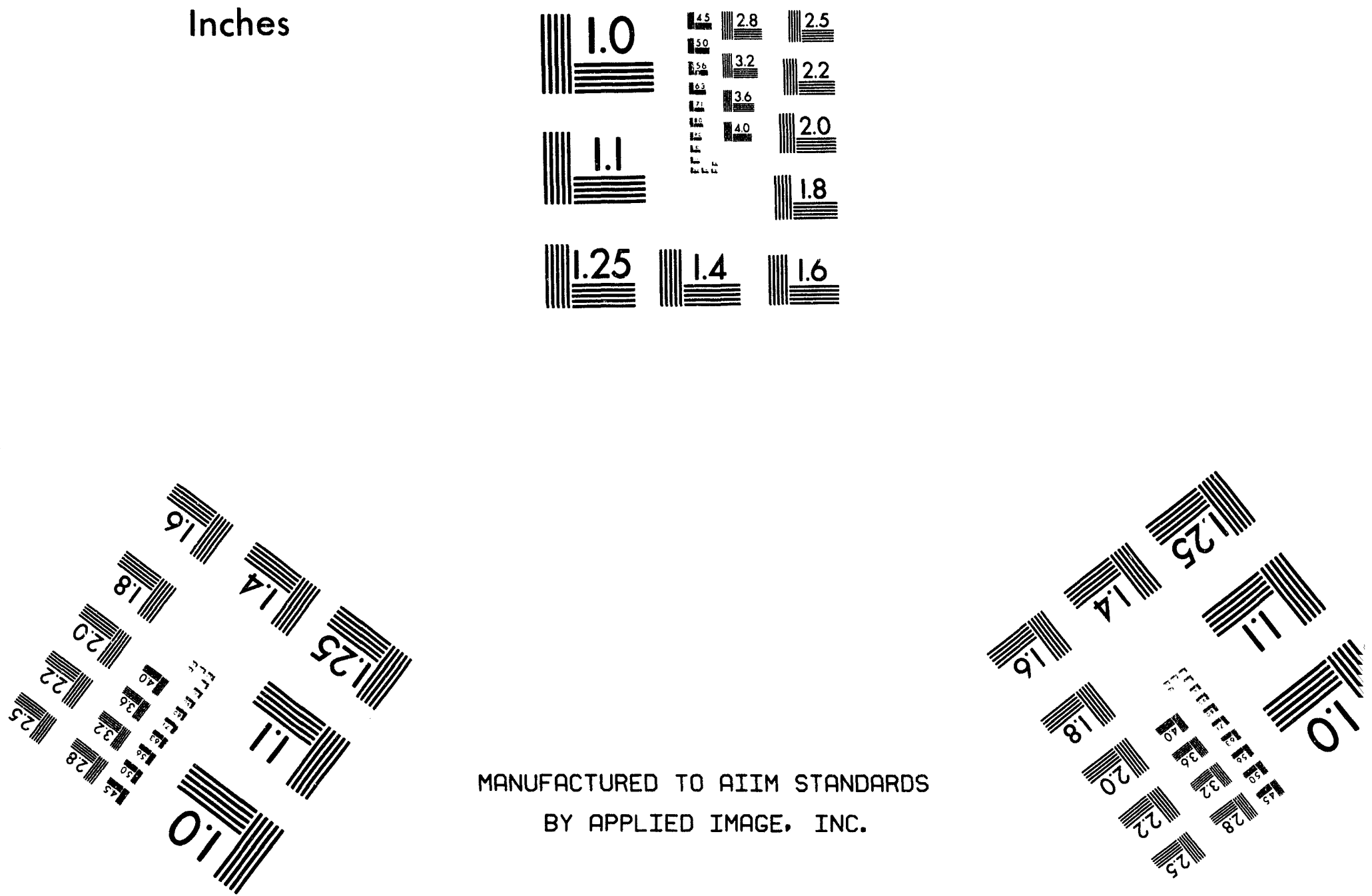




\section{EXPERIMENTAL CHARACTERIZATION OF ALS UNDULATOR RADIATION}

P.Heimann, D. Mossessian, A. Warwick, E. Gullikson*, C. Wang, S. Marks, H. Padmore and B. Kincaid

Advanced Light Source Accelerator and Fusion Research Division

Lawrence Berkeley Laboratory

University of California

Berkeley,CA 94720

* Center for X-ray Optics

Materials Sciences Division

Lawrence Berkeley Laboratory

University of California

Berkeley,CA 94720

July 15, 1994

"This work was supported by the Director, Office of Energy Research, Office of Basic Energy Sciences, Materials Sciences Division of the U.S. Department of Energy, under Contract no. DE-AC03-76SF00098 


\title{
Experimental Characterization of ALS Undulator Radiation.
}

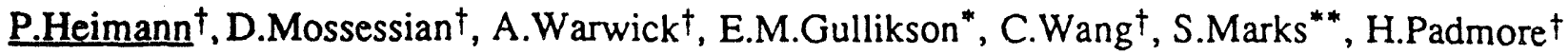 \\ and B.Kincaid ${ }^{\dagger}$. \\ †Advanced Light Source, Accelerator and Fusion Research Division \\ *Center for X-Ray Optics, Materials Sciences Division \\ ${ }^{* *}$ Engineering Division \\ Lawrence Berkeley Laboratory, Berkeley, CA 94720
}

\begin{abstract}
The radiation from the $5 \mathrm{~cm}$ period undulator at the Advanced Light Source (ALS) has been characterized using a transmission grating spectrometer. Spectral and angular distributions of radiation were measured for deflection parameter $K$ values between 0.45 and 2.12 at low storage ring current $(0.1-0.5 \mathrm{~mA})$. From the calibration of the spectrometer, the absolute flux density of the undulator harmonics has been determined together with the spectral linewidth. The electron beam emittance was determined by analyzing the angular distribution of the red-shifted fundamental. Comparison has been made with radiation calculations based upon the measured magnetic field data of the undulator. Including field errors, electron beam emittance and energy spread, good agreement is found between theoretically and experimentally determined harmonic widths and peak brightness.
\end{abstract}




\section{Introduction}

Radiation measurements are described from the first $5 \mathrm{~cm}$ period undulator installed in the Advanced Light Source storage ring. The U5.0 undulator is a permanent magnet-steel hybrid design with 89 periods and 4.5 meter length. Combined with a spherical grating monochromator the undulator covers the photon energy range from 80 to $1200 \mathrm{eV}$. However, the present undulator vacuum chamber limits the gap to a minimum value of $23 \mathrm{~mm}(K=2.12)$, corresponding to a minimum fundamental energy of $133 \mathrm{eV}$.

A transmission grating spectrometer (TGS) [1] was constructed in order to characterize the radiation from the undulators at the ALS and provide a confirmation of the undulators performance. The spectrometer was designed to measure the absolute intensities and spectral linewidths of the undulator harmonics as well as the angular distribution of undulator radiation. Thus, the results of the measurements can be compared with radiation calculations and the accuracy of the computer codes can be tested. The design of new undulators relies upon these calculations. Finally, determining the absolute flux from an insertion device provides the starting point for a quantitative evaluation of the throughput of a beamline.

For the U5.0 undulator the measurements were performed at four undulator gaps, for which the undulator magnetic field had been previously measured [2]. Since the undulator beamline utilizes the central cone of the $1 \mathrm{st}$, 3rd and 5th harmonics of undulator radiation, the intensity and linewidth of these harmonics were used as a test standard for the undulator performance. The results of the measurements were compared with radiation calculations, which include the measured magnetic field, emittance and energy spread of the electron beam. Angular distributions were measured showing the central cone and the red-shifted radiation off axis. The electron beam emittance at low current was determined from the angular distribution of the redshifted fundamental.

\section{Experimental}

A detailed description of the design and calibration of the spectrometer has been presented previously [1]. In a design similar to the spectrometers of Moltor and Ulm [3] and Tatchyn et al. [4], the TGS consists of a spherical focusing mirror, two interchangeable gold transmission gratings, and Si n on p photodiode behind a $5 \mu \mathrm{m}$ slit. The gratings have $2000 \AA$ and $5000 \AA$ periods and are lying on $150 \mu \mathrm{m}$ by $3.7 \mu \mathrm{m}$ and $17 \mu \mathrm{m}$ by $17 \mu \mathrm{m}$ support structure respectively. In these measurements the $2000 \AA$ period grating was mainly used because of its higher resolution. The theoretical spectral resolving power of the TGS, $\lambda \Delta \lambda \geq 500$, is derived from the 
width of the detector slit, the width of the mirror focus and the grating diffraction limit $\lambda \Delta \lambda=N$, where $N$ is the number of illuminated grating bars.

During the measurements the TGS was installed after the U5.0 front end at a distance of $15.5 \mathrm{~m}$ from the center of the undulator. The angular resolution of the TGS is determined by the size of two interchangeable pinholes in front of the grating, $300 \mu \mathrm{m}$ and $150 \mu \mathrm{m}$ in diameter, which set the angular acceptance of $20 \mu \mathrm{rad}$ and $10 \mu \mathrm{rad}$ respectively. The TGS vacuum chamber is mounted on a carriage in order that the TGS may be translated vertically and horizontally perpendicular to the undulator axis. The efficiencies of the TGS optical elements were determined to an accuracy of better than $10 \%$. These calibration measurements were performed using two reflectometers based on a laser produced plasma source and on an $\mathrm{x}$-ray tube with interchangeable anodes $[5,6]$.

In order to protect the gratings from damage, the radiation power density on the gratings was limited to $0.1 \mathrm{~W} / \mathrm{cm}^{2}$ during the undulator radiation measurements. This power restriction translated into a storage ring current between 0.1 and $0.5 \mathrm{~mA}$ depending on the undulator gap.

The measurements were performed in two ways. Undulator radiation spectra were recorded by scanning the detector for a fixed position of the grating pinhole relative to the undulator axis. Angular distributions of radiation were obtained by translating the spectrometer with constant photon energy (fixed detector position). For an angular scan in the vertical direction, the position of the detector was corrected for the change in the incidence angle on the grating.

The spectral resolution of the TGS was verified by measuring the absorption line of a $\mathrm{LaF}_{3}$ thin film. $\mathrm{LaF}_{3}$ has a resonance, $3 \mathrm{~d}_{5 / 2}-4 \mathrm{f}$, at $834.3 \mathrm{eV}$ photon energy with a natural linewidth of about $0.5 \mathrm{eV}$ FWHM [7]. The filter, $\sim 200 \AA$ of $\mathrm{LaF}_{3}$ evaporated on an $\mathrm{Al}$ foil, was inserted in the photon beam in front of the TGS, and the M5 absorption line was recorded within the lineshape of the undulator 5 th harmonic. The resolving power of the spectrometer, $\lambda \Delta \Delta \lambda$, was determined to be 200 for the first order of diffraction and 630 for the fourth order. The actual resolution was poorer than it was expected because of the diffraction from the support structure of the transmission grating. However, the resolution in the fourth order of diffraction was sufficient to determine the spectral width of the U5.0 fifth harmonic without resorting to deconvolution techniques.

The wavelengths of the $\mathrm{LaF}_{3} 3 \mathrm{~d}_{5 / 2}-4 \mathrm{f}$ resonance and of the $\mathrm{Al} 1 \mathrm{~s}$ absorption edge were used to verify the wavelength scale of the TGS. To calibrate the wavelengths of the undulator harmonics at all gaps, the electron beam energy was fit with the measured wavelengths according to the equation

$$
\lambda_{\mathrm{n}}=\frac{\lambda_{\mathrm{u}}}{2 n \gamma^{2}} \times\left(1+K^{2} / 2\right)
$$

where $\lambda_{n}$ is the wavelength of the $n$th undulator harmonic, $\lambda_{u}$ is the undulator period, $\gamma$ is proportional to the electron beam energy and $K$ is the undulator deflection parameter. $K$ was 
derived from the measured magnetic field. The calculated electron beam energy $E=1.515 \mathrm{GeV}$ is in a good agreement with the value of $1.520 \pm 0.005$ measured by accelerator physics group.

\section{Angular distribution and electron beam emittance}

The radiation of an undulator harmonic is concentrated in a narrow central cone, whose width is determined by the harmonic wavelength, electron beam emittance, and electron beam energy spread. Since the dispersion function along the straight sections of the ALS storage ring is equal to zero, the energy spread does not affect the angular characteristics of the undulator radiation. In a single electron approximation the rms angular width of the central cone can be estimated using the expression [8]

$$
\sigma_{r^{\prime}}=\sqrt{\frac{\lambda_{n}}{2 L}}
$$

where $\lambda_{n}$ is the wavelength of the $n$th harmonic and $L$ is the length of the insertion device. At the spectrometer pinhole at a distance $D$ from the center of the undulator, the emittance will contribute to the size of the central cone following:

$$
\sigma_{x^{\prime}, y^{\prime}}=\sqrt{\sigma_{r^{\prime}}^{2}+\left(\sigma_{x^{\prime}, y^{2}}\right)^{2}+\left(\sigma_{x, y^{\mathrm{e}}}^{\mathrm{e} / D)^{2}}\right.}
$$

where $\sigma_{x, y^{\mathrm{e}}}$ and $\sigma_{x^{\prime}, y^{\perp}}$ are the horizontal $(\mathrm{x})$ or vertical $(\mathrm{y}) \mathrm{rms}$ size and divergence of the electron beam.

In Fig. 1 are displayed horizontal and vertical scans through the angular distribution at a fundamental wavelength of $31.1 \AA(K=0.45)$. In the horizontal plane, where the radiative width $\sigma_{\mathbf{r}^{\prime}}$ is smaller than the emittance width, the shape of the angular distribution is gaussian. The measured ms width of the horizontal distribution, $\sigma_{x^{\prime}}=31 \mu \mathrm{rad}$, is in a good agreement with the width estimated using Eq.(3). Since the vertical emittance is much smaller than the natural width $\sigma_{\mathrm{r}^{\prime}}$ of the central cone, the shape of the vertical distribution is non-gaussian, close to the shape in the single electron approximation. In this case the $\sigma_{y^{\prime}}=21 \mu \mathrm{rad}$, calculated using Eq.(3) agrees worse with the measured width of $25 \mu \mathrm{rad}$ ms. The dashed curves represent a calculation using the URGENT code [9], which includes emittance and successfully reproduces the measurements.

As the angle $\theta$ to the undulator axis increases the wavelength of the harmonics changes following the equation

$$
\lambda_{\mathrm{n}}=\frac{1}{n} \frac{\lambda_{u}}{2 \gamma^{2}}\left(1+\frac{K^{2}}{2}+\gamma^{2} \theta^{2}\right)
$$

The angular distribution of the red-shifted harmonic has a form of a ring. Neglecting emittance, the width of the ring can be estimated by assuming that over the length of the undulator the difference 
in angle $\Delta \theta$ produces an extra path difference of $\lambda$, which results in net destructive interference [10]. In this case

$$
\Delta \theta \approx-\theta+\sqrt{\theta^{2}+\frac{2 \lambda_{n}}{L}}
$$

where $\lambda_{n}$ is the wavelength at the angle $\theta$. It can be seen from equation (5) that the angular width $\Delta \theta$ is a decreasing function of $\theta$. Another contribution to the $\Delta \theta$ is the near field effect, which results from observing different parts of the undulator at different angles. Near field effect has an opposite dependence increasing with $\theta$.

The measured angular distribution of the red-shifted fundamental is shown in Fig.2. The undulator $K$ parameter was 0.45 . The distribution was recorded by tuning the spectrometer to a $\lambda$ $1.7 \AA$ larger than the first harmonic wavelength on axis and by translating the spectrometer horizontally and vertically. The angular width of the ring pattern calculated using formulae (5) is $8.5 \mu \mathrm{rad}$. Because of the electron beam emittance, the measured FWHM are considerably wider, $58 \mu \mathrm{rad}$ in the horizontal and $27 \mu \mathrm{rad}$ in the vertical directions.

The angular distribution of the off-axis undulator radiation is a sensitive measure of the emittance because of the small intrinsic width. Also, in contrast to the harmonic peak intensities, orthogonal cuts through the angular distribution depend separately on the horizontal and vertical emittances. In figure 2 are displayed calculations with URGENT, which include the emittance parameters giving the best agreement with the measured curves. For the ALS in low current operation, the electron beam emittance is derived to be $\varepsilon_{x}=4.4 \times 10^{-9} \mathrm{~m} \times \mathrm{rad}$ and $\varepsilon_{y}=2.0 \times 10^{-10} \mathrm{~m} \times \mathrm{rad}$. Theoretical values for horizontal and vertical beta functions were assumed. In the vertical direction, where the angular width is comparable to the grating pinhole acceptance, a numerical deconvolution was made for the measured angular distribution. The calculated vertical emittance should still be considered as an upper limit because of the contribution from the near field effect and the spectrometer resolution. The measured ALS horizontal emittance agrees with the theoretical value for $\varepsilon_{x}$ and shows less than $10 \%$ horizontal-vertical emittance coupling.

\section{U5.0 on-axis spectra}

The spectrum produced by a charged particle moving through an undulator consists of discrete harmonics. The wavelength $\lambda_{n}$ of $n$th harmonic is determined by the equation (1) and depends on the electron beam energy $E$ and the undulator deflection parameter $K$. In the approximation of a single electron and a sinusoidal magnetic field, the width of a harmonic is inversely proportional to the number of undulator magnetic periods $N, \Delta \lambda / \lambda=1 / n N$. The electron beam emittance and energy spread as well as the undulator magnetic field errors may all broaden the width and lower the peak brightness of the harmonics. To analyze these effects, a quantitative 
comparison has been made between measured and calculated on-axis harmonic intensities and spectral widths.

Two spectra of U5.0 undulator radiation on axis are presented in Fig.3. The on-axis position of the spectrometer was determined from the angular distribution of the 5th harmonic central cone. As it was discussed above, the angular size of the grating aperture is always smaller than the width of the radiation central cone. Thus, the recorded wavelength distribution is not affected by the aperture angular acceptance and can be used to determine the on-axis brightness and width of the harmonics. These spectra show examples of medium and low $K$ values. For $K=$ 2.12 , there are 27 harmonics observed, while for $K=0.45$ the fundamental is dominant. In the tails of the fundamental peaks, especially for $K$ of 0.45 , oscillations are visible, caused by the finite number of periods in the undulator. The measured spectra contain added complexity because of the higher orders of diffraction of the transmission grating.

The calculations of the undulator radiation spectra are based on a computer program URGENT [9], which includes the electron beam emittance, but not energy spread or real magnetic field. For the emittance, values derived above from angular distribution measurements were used, $\varepsilon_{x}=4.4 \times 10^{-9} \mathrm{~m} \times \mathrm{rad}$ and $\varepsilon_{y}=2.0 \times 10^{-10} \mathrm{~m} \times \mathrm{rad}$. The energy spread was included by convoluting the calculated lineshape with a Gaussian function whose $\Delta \lambda$ was set by differentiating the equation (1) with respect to the beam energy. The theoretical value of $6.5 \times 10^{-4}$ was employed for the electron beam energy spread $\Delta E / E$. The effect of the real magnetic field was calculated separately with a computer code, developed by B.Kincaid [2]. This code starts from the measured magnetic field data and derives corrections to the ideal harmonic areas and peak heights. After the energy spread convolution, the URGENT calculations were multiplied by the area correction factor for the magnetic field, which implies a small error in the harmonic peak height.

Fig. 4 shows the measured and calculated 1st, 3rd and 5th harmonics for the same $K$ values, 2.12 and 0.45 , as Fig.3. The flux density (photons $/ \mathrm{sec} / \mathrm{mrad}^{2} / 0.1 \% \mathrm{bw}$ ) of the harmonics was derived from the detector current by using the measured efficiencies of the TGS optical elements, the angular acceptance of the grating pinhole and the width of the detector slit. Overlapping contributions of higher orders of diffraction were taken into account. The measured and calculated harmonics agree well in the spectral width and lineshape. However, the quantitative comparison between the measured and calculated peak flux densities is not as good. The difference in the absolute intensities is attributed to errors in the spectrometer calibration and in the electron beam current. For $K$ of 2.12 with a current of $0.1 \mathrm{~mA}$, there is a possible $25 \%$ error in the beam current measurements. For the fundamental, the width $\Delta \lambda / \lambda \approx 1 / 80$ approaches the ideal value $1 / 89$ confirming the combined performance of the ALS as a low emittance storage ring and of the U5.0 undulator. The higher harmonics have increasing sensitivity to the factors of emittance, energy spread and field errors because of their decreasing spectral and angular widths. The 
measured linewidth of the 5 th harmonic, $\Delta \lambda \lambda \approx 1 / 200$, is significantly larger than the ideal width, 1/445. The calculated 5th harmonic width contains about equal contributions from the emittance and energy spread with field errors having a small effect.

\section{Conclusion}

The radiation from the ALS U5.0 undulator has been characterized with a transmission grating spectrometer. The wavelengths of the undulator harmonics were calibrated from the spectrometer wavelength scale. The first harmonic central cone was observed to be Gaussian in the horizontal direction because of emittance, but non-Gaussian in the vertical where the emittance contribution is small. From the angular distribution of the red-shifted fundamental, the ALS emittance at low current was shown to be in agreement with the theoretical $\varepsilon_{x}$ and less than $10 \%$ coupling. The first harmonic had a nearly ideal lineshape, while the 5 th harmonic was broadened by emittance and energy spread. After including energy spread and magnetic field errors, calculations with URGENT were able to reproduce the measured harmonic peak flux densities and lineshapes.

\section{Acknowledgment}

This work is supported by Director, Office of Energy Research, Office of Basic Energy Sciences, Materials Sciences Division of the U.S. Department of Energy under Contract Number DE-AC03-76SF00098. 


\section{References}

1. D.A. Mossessian, P.A. Heimann, E.Gullikson, R.K. Kaza, J. Chin, J. Akre. To be published in Nuclear Instruments and Methods in Physics Research.

2. S.Marks, D.Humphries, B.Kincaid, R.Schlueter, C.Wang. SPIE Proceedings, Vol. 2013, 171-181

3. K.Molter and G.Ulm. Rev. Sci. Instrum. 63, 1296 (1992)

4. R.Tatchyn, E.Kallne, A.Toor, T.Cremer and P.Csonka. Rev. Sci. Instrum. 60, 1579 (1989).

5. E.M.Gullikson, J.H.Underwood, P.C.Batson, and V.Nikitin. Journal of X-ray Science and Technology $\underline{3}, 283$ (1992)

6. B.L.Henke and M.A.Tester. Advances in X-ray Analysis, Vol. 18, p.76 (Plenum Press, 1975)

7. G.van der Laan and B.T.Thole. Joumal of electron spectroscopy and related phenomena, 46 (1988), 123-129.

8. Kwang-Je Kim. AIP Conferences Proceedings, 184 , American Institute of Physics, New York (1989)

9.R.P.Walker and B.Diviacco. Rev.Sci.Instrum. 63 (1), 392-395, January 1992.

10. R.Walker. Nuclear Instruments and Methods in Physics Research, A267 (1988) 537-546 


\section{Figure Captions}

Figure 1: The angular distribution of the 1 st harmonic for $\mathrm{K}=0.45$. The solid curves represent measurements and the dashed curves calculations with URGENT, including the emittance.

Figure 2: The angular distribution of the red-shifted fundamental for $\mathrm{K}=0.45$ and with a wavelength shift of $1.7 \AA$ from the fundamental $\lambda=31.1 \AA$. The solid curves display measurements and the dashed curves calculations with URGENT, including the emittance.

Figure 3: On axis spectra of the U5.0 undulator radiation for $\mathrm{K}$ of 0.45 and 2.12. The uncorrected detector current is proportional to the power density of the harmonics.

Figure 4: The measured and calculated odd harmonics for $\mathrm{K}$ of 0.45 and 2.12. The solid curves show measurements and the dashed curves calculations with URGENT, including emittance and energy spread. The flux densities are corrected for the effect of a real magnetic field and normalized to $400 \mathrm{~mA}$ electron beam current. 

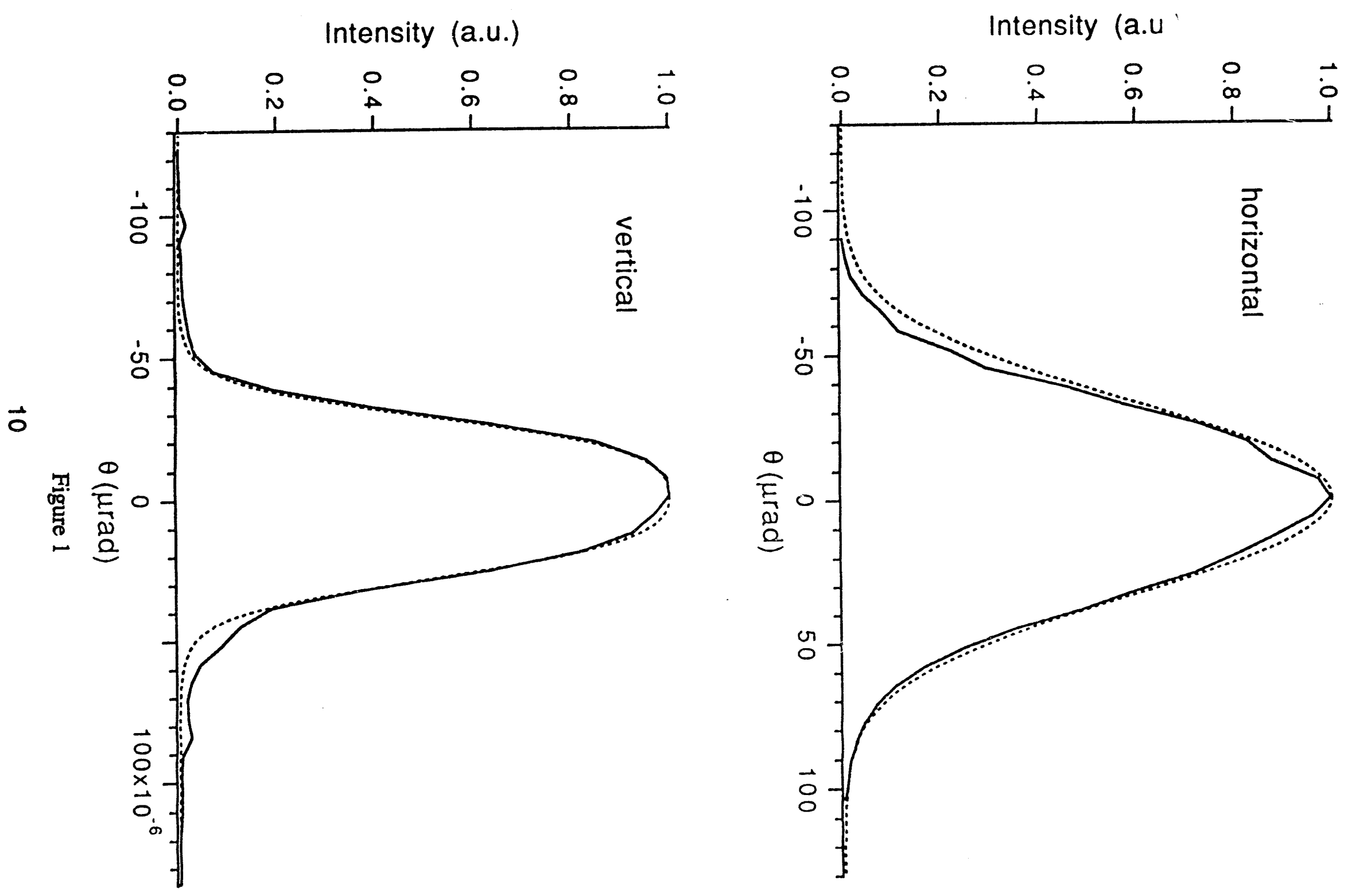

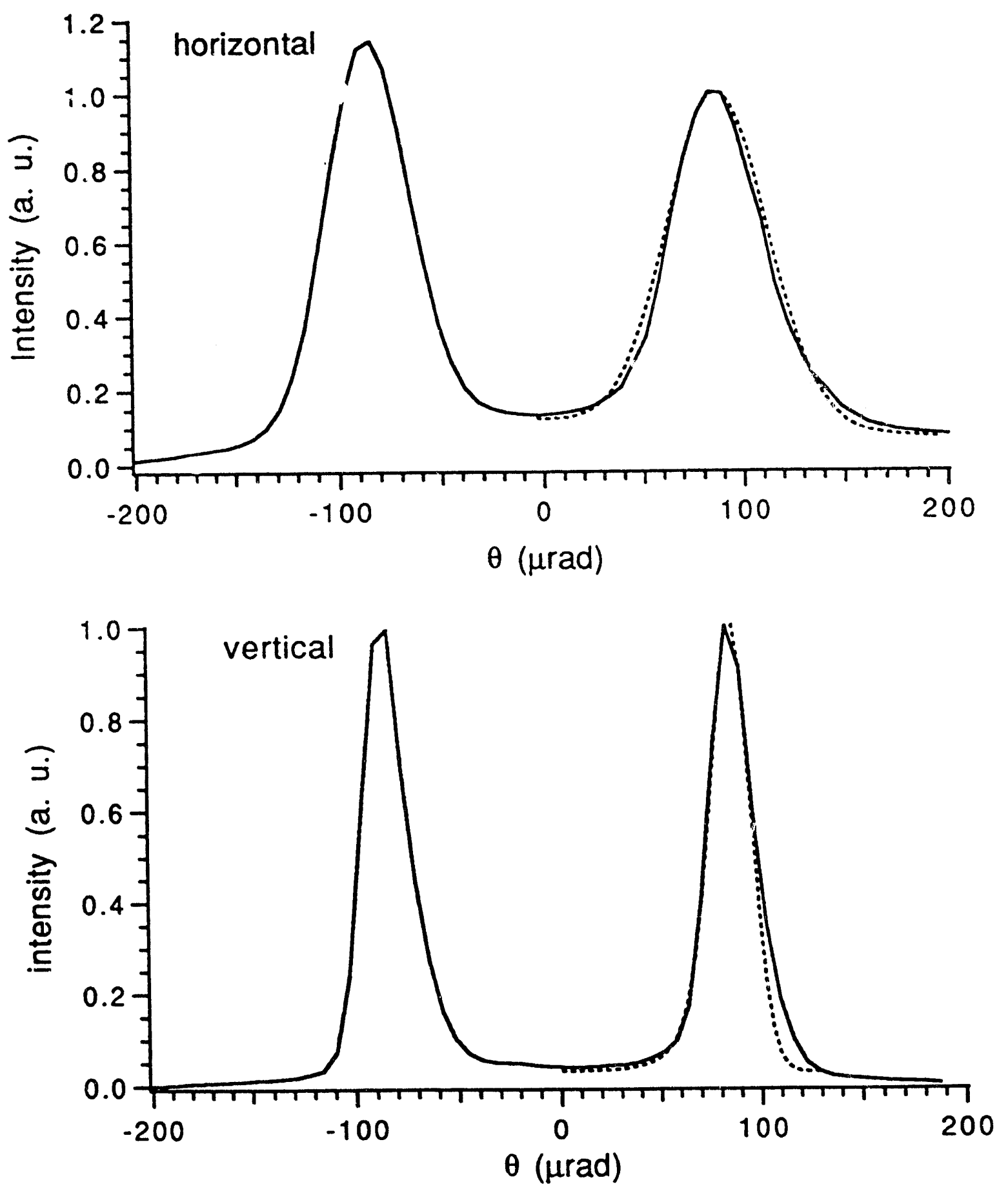

Figure 2 

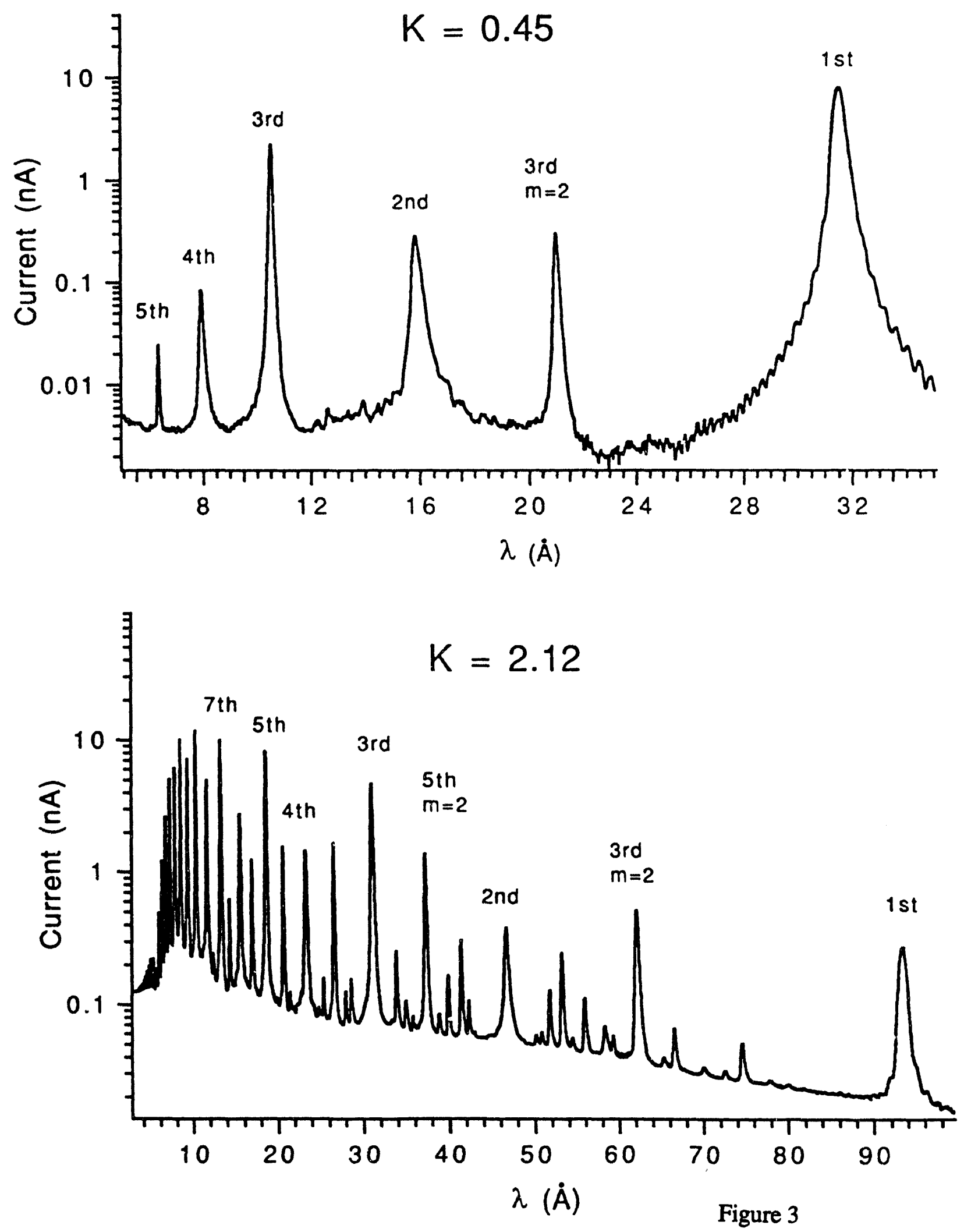


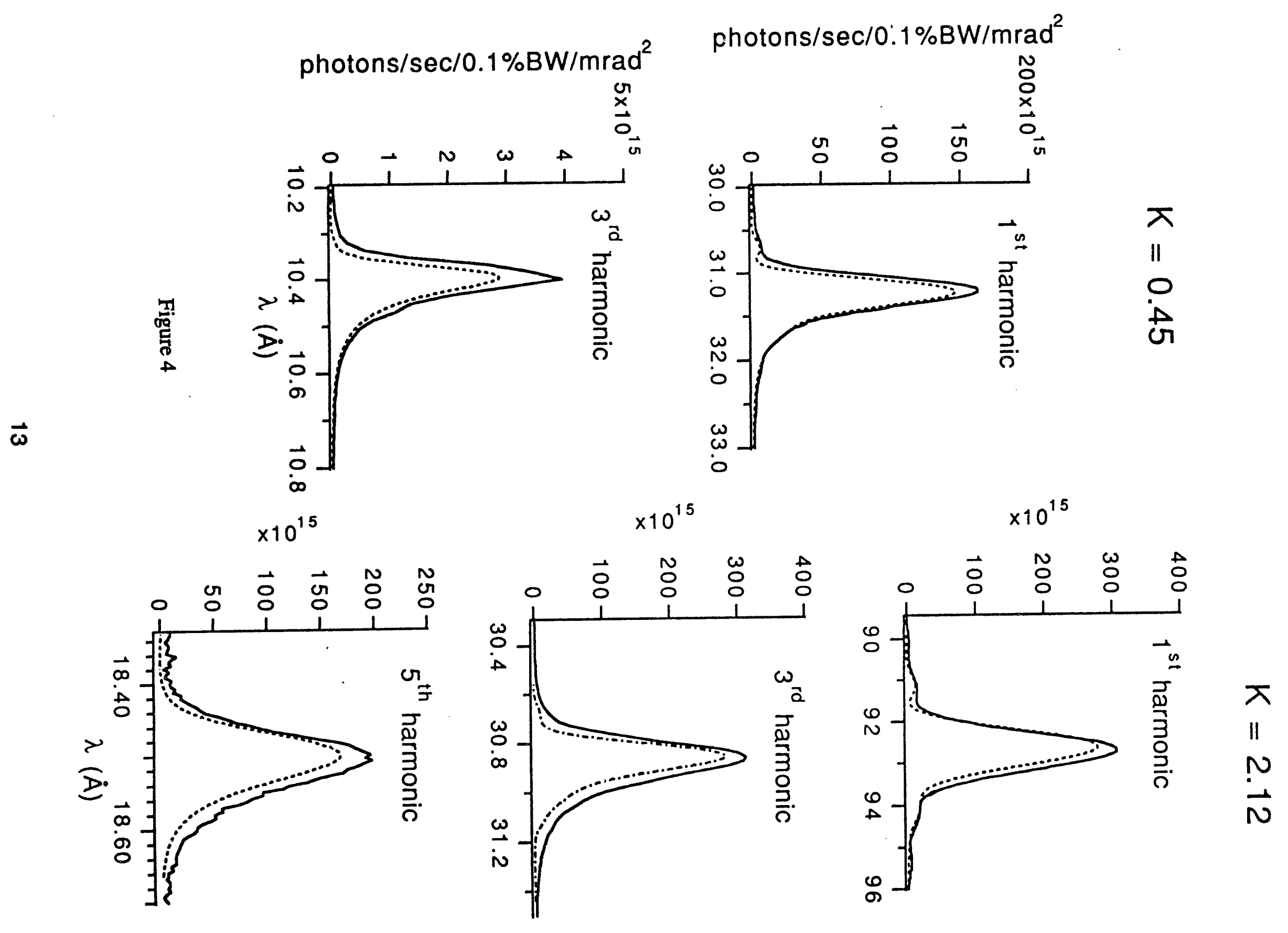



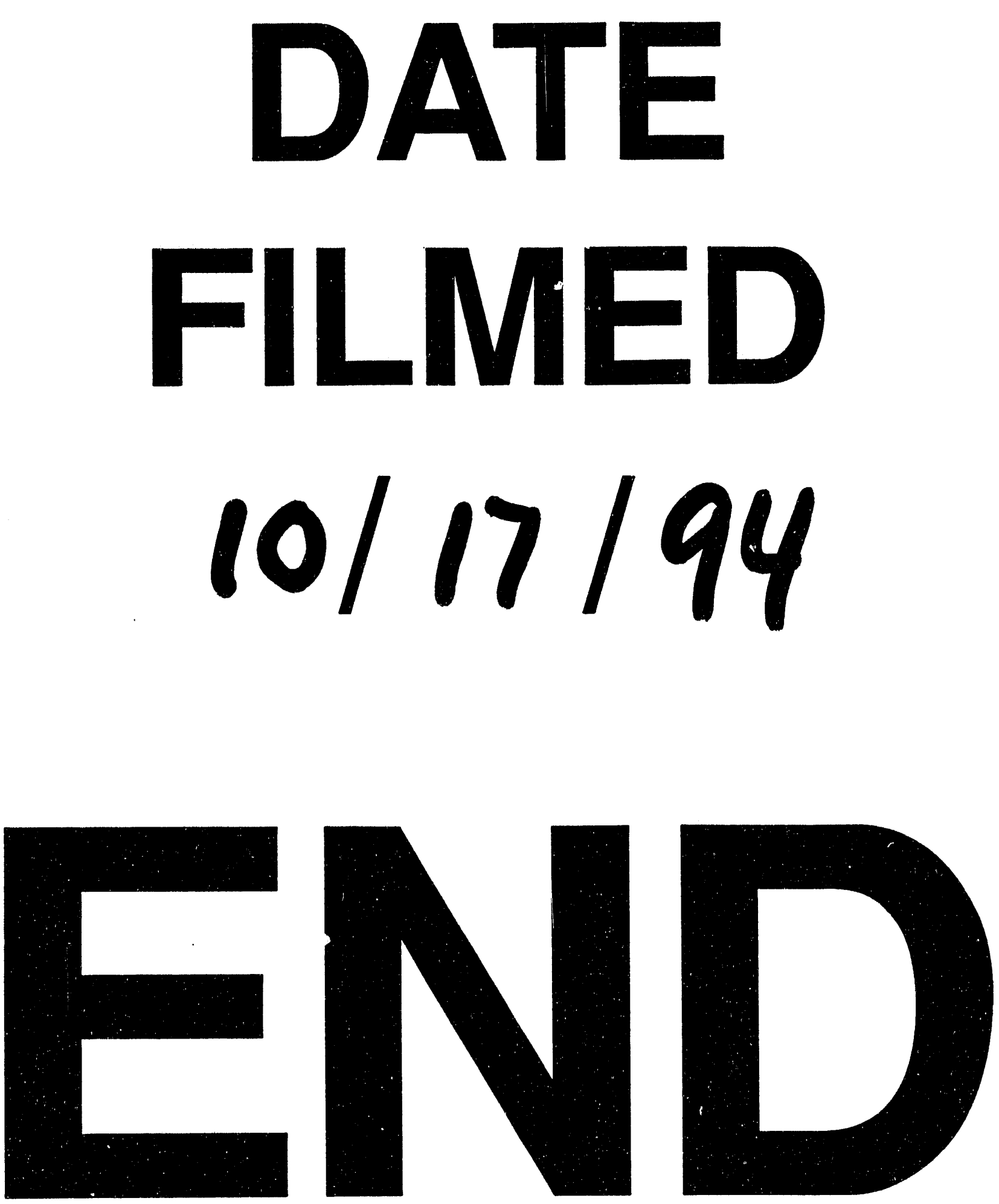
